\title{
Review of Phase II Basket Trials for Precision Medicine
}

\author{
Yeonhee Park* \\ Department of Public Health Sciences, Medical University of South Carolina, USA
}

*Corresponding author: Yeonhee Park, Department of Public Health Sciences, Medical

Received Date: April 22, 2019

University of South Carolina, USA.

\author{
Published Date: May 02, 2019
}

\begin{abstract}
The advancement of molecular biology and genomic technologies has made paradigm shift from conventional paradigm with cytotoxic agents to precision medicine using molecularly targeted agents. The premise of precision medicine is that only a subset of patients is sensitive to and benefits from a given treatment. Under this new paradigm for clinical development, studies for drug activity require to consider inter-patient heterogeneity and intra-patient heterogeneity. Basket trials evaluate the effect of the targeted therapy with a certain genetic mutation across cancer types. The basket study is increasingly becoming recognized as an attractive approach for drug development under the precision medicine paradigm. It leads to novel statistical methodologies development and conducting clinical trials based on the basket study. In this review paper, we describe the basket trials with statistical methods and examples.
\end{abstract}

Keywords: Basket trials; Master protocols; Phase II trials; Precision medicine

\section{Introduction}

Traditionally, clinical trials have focused on the treatment for patients classified by a certain site of cancer in the body and stage. With the tremendous advancement of molecular biology and genomic technologies, cancer cell growth and progression can be defined at cellular and molecular levels, and treatments have been developed with presence or absence of molecular markers or genetic mutations at a particular site. For example, trastuzumab works effectively only in patients with a breast tumor that expresses high levels of HER-2, and erlotinib is only effective for treating patients with non-small cell lung cancer that over-expresses the epidermal growth factor receptor. In an era of precision medicine, one-sizefit-all medicine does not work well, and individual variability in genes and environment for each person or subgroup should be considered for drug development. The precision medicine clinical trials with the targeted therapies toward specific molecular marker or genetic mutation are promising to improve clinical outcomes as well as understanding of the treatment and provide actionable guidance for the confirmatory trials or drug marketing. Here, we focused on phase II basket trials for precision medicine, which is exploratory to find a signal demonstrating statistical significance of therapeutic efficacy. We briefly describe phase II basket trials. We review statistical methods and provide real trial examples for basket trials.

\section{Basket Trials}

Basket trials evaluate one therapy targeting a certain genetic mutation on multiple diseases or multiple disease subtypes. In other words, patients in basket studies have a certain genetic mutation in common regardless of the site of cancer in the body. The grouped diseases or tumor types form baskets, and substudies are conducted for single-arm, phase II trials on populations by tumor groups within the basket. Specifically, baskets can be specified by disease, disease-mutation, or disease-drug-mutation. For example, original study B2225 enrolled non-melanoma BRAF V600E mutation-positive patients with 40 different solid tumors or hematologic malignancies (i.e., disease-specific baskets) to evaluate the efficacy of imatinib, which is an oral inhibitor of BRAF [1]; CREATE trial [2] evaluates the efficacy of crizotinib which inhibits multiple oncokinases including mesenchymal-epithelial transaction factor and anaplastic lymphoma kinase (i.e., diseasemutation-specific baskets); And CUSTOM trial [3] simultaneously evaluates the five targeted therapies by genetic mutation and each treatment is evaluated in three different diseases (i.e., 15 diseasedrug-mutation-specific baskets).

Phase 2 basket trials discover the drug activity early against cancer types for patients with the defined genetic mutation rather than perform hypothesis testing. The promising results from some cancer types where the targeted agent is active should be confirmed in an expanded phase 2 trials and allow us to learn for a phase 3 study. Basket studies will evaluate the efficacy of targeting a specific genetic mutation by borrowing the strength of data combining the baskets which are linked together. They evaluate multiple disease simultaneously and would accelerate the drug development. Moreover, basket trials can include rare disease subtypes within 
the basket. However, there are some challenges of basket trials: (1) they may have small sample size for rare genetic mutation; (2) a particular genetic mutation is often uncertain to be considered in a tumor of a particular histology type, e.g., vemurafenib works effectively for non-melanoma BRAF V600E mutation-positive cancers but not for analogous colorectal cancer [4-6]; (3) there could be multiple genomic variants for a given gene, which might affect response to the therapy differently across tumor types.

\section{Statistical Methods}

We review some statistical methodologies developed for basket trials. The simple and straightforward approach is the pooled analysis, which pools data across cancer types by assuming the patients with different types of cancer are homogeneous. So, it would yield a bias if treatment effect is heterogeneous across cancer types. Another straightforward approach is to conduct parallel trials in each basket independently. But this approach is less efficient and might not be feasible because it would require more total sample size. To improve the efficiency for basket trials, borrowing information across cancer types is essential. LeBlanc et al. [7] and Cunanan et al. [8] propose a strategy using Simon's two-stage design. LeBlanc et al. [7] conducts futility analysis based on the pooled data of all cancer types to terminate the entire trial and additional futility analysis is conducted for each cancer type. In Cunanan et al. [8], the heterogeneity of treatment effect is evaluated across baskets in the first stage, and then separately evaluated for the remaining baskets where the targeted agent is effective. Simple Bayesian approach shrinks the treatment effect of cancer types towards the mean of the prior distribution with a fixed spread. A hierarchical Bayesian approach treats the mean and variance of prior distribution at random quantities assuming that the treatment effects of cancer types are exchangeable and are centered at common mean [9-11]. However, these approaches could fail to borrow information across cancer types with 10 or fewer cancer subtypes and are more likely to inflate type I error rate [12]. The hierarchical Bayesian approach is improved by either using a shrinkage prior defined as a function of a similarity measure of the treatment effect across cancer types [13], or aggregating cancer types into latent subgroups based on the longitudinal biomarker measurements [14]. Another way to discover the cancer types in which the targeted agent is active is to use Bayesian model averaging to model potential information borrowing $[15,16]$. This approach borrows information across cancer types when the data indicates that the response probabilities in the cancer type are heterogeneous and the drug activity in different type are correlated. To avoid borrowing information from extreme cancer type, Neuenschwander et al. [17] proposes exchangeability/non-exchangeability approach as a robust mixture extension unlike the standard hierarchical Bayesian model assuming full exchangeability for parameter of cancer type. Hobb and Landin [18] proposes method to monitor exchangeability of cancer types at interims of basket trials. They address, by accommodating the treatment heterogeneity across cancer types, one of challenges of basket trials for BRAF-V600 study showing the vemurafenib is effective in treating melanoma with BRAF
V600E mutation but BRAF V600E mutation-positive patients with colorectal cancer don't benefit from vemurafenib. In addition, they control type I error rate better. Bayesian baskets using predictive biomarkers are proposed to randomly assign patients to treatments through a Bayesian adaptive randomization and compared with the conventional basket design [19].

\section{Examples}

As of July 2018, there are 27 basket trials found on ClinicalTrials. gov for intervention study in solid tumors [20]. Original basket trial study was the study B2225 evaluating the efficacy of imatinib [1]. The study was categorized as a basket trial by Woodcock and LaVange [21]. Also, vemurafenib is the first targeted therapy based on the basket trial approved by FDA for the treatment of patients with BRAF V600E genetic mutant Erdheim-Chester Disease [22]. The SHIVA trial is one of completed basket trials which does not show a promising outcome of the molecular targeted agent. It is a randomized phase II study compared molecularly targeted therapy with conventional therapy in patients with refractory cancer. NCIMATCH trial [23-25] and the signature program developed by Slosberg et al. [26] consist of several phase II basket protocols. Each basket trial aims to evaluate a targeted agent in patients with different histologies and an actionable mutation. They are efficient to find signal more quickly across multiple tumors and multiple targeted agents. More examples are described in [21,27,20]. Hazim and Prasad [28] provides a systematic overview of published basket trial with the tumor histology and the response rate based on a search Google scholar for the terms cancer and basket trial on March 31, 2018.

\section{Discussion and Conclusion}

We have reviewed phase II basket trials for precision medicine. To address challenges of basket trials, we need to take care of indication selection. In particular, for the application with rare genetic mutation, extensive cooperation is required to open trials globally. The novel statistical designs for basket trials have been proposed, but it is still challenging to design efficient basket trials with recent tremendous development of genomic profiling technologies and next generation sequencing. The basket trial designs can be more complicated to have the baskets for more than one genetic mutation or to evaluate multiple targeted drugs for several genetic mutations and tumor types. Other types of a master protocol, umbrella and platform trials, can be incorporated to design trials. The innovative strategies for basket trials should be developed to expedite the drug development process and to treat target patients who are likely to benefit with the targeted agents in an era of precision medicine.

\section{Acknowledgement}

The work is supported in part by Biostatistics Shared Resource, Hollings Cancer Center, Medical University of South Carolina (P30 CA138313)

\section{Conflict of Interest}

No conflict of interest. 


\section{References}

1. Heinrich MC1, Joensuu H, Demetri GD, Corless CL, Apperley J, et al. (2008) Phase II, open-label study evaluating the activity of imatinib in treating life-threatening malignancies known to be associated with imatinib-sensitive tyrosine kinases. Clin Cancer Res 14(9): 2717-2725.

2. Schöffski P, Wozniak A, Stacchiotti S, Rutkowski P, Blay JY, et al. (2017) Activity and safety of crizotinib in patients with advanced clear-cell sarcoma with MET alterations: European Organization for Research and Treatment of Cancer phase II trial 90101 'CREATE'. Ann Oncol 28(12): 3000-3008.

3. Lopez-Chavez A, Thomas A, Rajan A, Raffeld M, Morrow B, et al. (2015) Molecular profiling and targeted therapy for advanced thoracic malignancies: a biomarker-derived, multiarm, multihistology phase II basket trial. Journal of clinical oncology 33(9): 1000

4. Kopetz S, J Desai, E Chan, JR Hecht, PJ O’Dwyer, et al. (2010) PLX4032 in metastatic colorectal cancer patients with mutant BRAF tumors. Journal of Clinical Oncology 28(15_suppl): 3534-3534.

5. Turski ML, Vidwans SJ, Janku F, Garrido-Laguna I, Munoz J, et al. (2016) Genomically driven tumors and actionability across histologies: BRAFmutant cancers as a paradigm. Mol Cancer Ther 15(4): 533-547.

6. Karoulia Z, E Gavathiotis, PI Poulikakos (2017) New perspectives for targeting RAF kinase in human cancer. Nat Rev Cancer 17(11): 676.

7. LeBlanc M, C Rankin, J Crowley (2009) Multiple histology phase II trials Clin Cancer Res 15(13): 4256-4262.

8. Cunanan KM, Iasonos A, Shen R, Begg CB, Gönen M (2017) An efficient basket trial design. Stat Med 36(10): 1568-1579.

9. Thall PF, Wathen JK, Bekele BN, Champlin RE, Baker LH, et al. (2003) Hierarchical Bayesian approaches to phase II trials in diseases with multiple subtypes. Stat Med 22(5): 763-780.

10. Berry SM, Broglio KR, Groshen S, Berry DA (2013) Bayesian hierarchical modeling of patient subpopulations: efficient designs of phase II oncology clinical trials. Clin Trials 10(5): 720-734.

11. Cunanan KM, Iasonos A, Shen R, Gönen M (2019) Variance prior specification for a basket trial design using Bayesian hierarchical modeling. Clinical Trials 16(2):142-153.

12. Freidlin B, EL Korn (2013) Borrowing information across subgroups in phase II trials: is it useful? Clin Cancer Res 19(6): 1326-1334.

13. Chu Y, Y Yuan (2018) A Bayesian basket trial design using a calibrated Bayesian hierarchical model. Clin Trials 15(2): 149-158.
14. Chu Y, Y Yuan (2018) BLAST: Bayesian latent subgroup design for basket trials accounting for patient heterogeneity. Journal of the Royal Statistical Society: Series C (Applied Statistics) 67(3): 723-740.

15. Simon R, Geyer S, Subramanian J, Roychowdhury S (2016) The Bayesian basket design for genomic variant-driven phase II trials. Semin Oncol 43(1): 13-18.

16. Liu R, Liu Z, Ghadessi M, Vonk R (2017) Increasing the efficiency of oncology basket trials using a Bayesian approach. Contemporary clinical trials 63: 67-72.

17. Neuenschwander B, Wandel S, Roychoudhury S, Bailey S (2016) Robust exchangeability designs for early phase clinical trials with multiple strata. Pharmaceutical statistics 15(2): 123-134

18. Hobbs BP, R Landin (2018) Bayesian basket trial design with exchangeability monitoring. Statistics in medicine 37(25): 3557-3572.

19. Trippa L, BM Alexander (2016) Bayesian baskets: a novel design for biomarker-based clinical trials. Journal of Clinical Oncology 68: 2864.

20. Janiaud P, S Serghiou, JP Ioannidis (2018) New clinical trial designs in the era of precision medicine: an overview of definitions, strengths, weaknesses, and current use in oncology. Cancer treatment reviews 73: $20-30$

21. Woodcock J, LM LaVange (2017) Master protocols to study multiple therapies, multiple diseases, or both. New England Journal of Medicine 377(1): 62-70.

22. Hyman DM (2015) Vemurafenib in multiple nonmelanoma cancers with BRAF V600 mutations. New England Journal of Medicine 373(8): 726 736.

23. Brower V (2015) NCI-MATCH pairs tumor mutations with matching drugs. Nat Biotechnol 33(8): 790-791.

24. McNeil C (2015) NCI-MATCH launch highlights new trial design in precision-medicine era. JNCI: Journal of the National Cancer Institute 107(7).

25. Do K, G O'Sullivan Coyne, AP Chen (2015) An overview of the NCI precision medicine trials-NCI MATCH and MPACT. Chinese clinical oncology 4(3): 31

26. Hirakawa A, Junichi Asano, Hiroyuki Sato, Satoshi Teramukaic (2018) Master protocol trials in oncology: Review and new trial designs. Contemporary clinical trials communications 12: 1-8.

27. Hazim A, V Prasad (2018) A pooled analysis of published, basket trials in cancer medicine. European Journal of Cancer 101: 244-250. 